\title{
Factor V Leiden and Prothrombin 20210A Mutations among Turkish Pediatric Leukemia Patients
}

\author{
Dilara Fatma Akın, ${ }^{1}$ Kadir Sipahi, ${ }^{1}$ Tuğba Kayaalp, ${ }^{1}$ Yonca Eğin, ${ }^{2}$ Serpil Taşdelen, ${ }^{1}$ \\ Emin Kürekçi, ${ }^{3}$ Üstün Ezer, ${ }^{1}$ and Nejat Akar ${ }^{4}$ \\ ${ }^{1}$ LOSEV the Foundation for Children with Leukemia, Ankara, Turkey \\ ${ }^{2}$ Department of Pediatric Genetics, Ankara University, Ankara, Turkey \\ ${ }^{3}$ Department of Pediatric Haematology, Gülhane Military Medical Academy, Ankara, Turkey \\ ${ }^{4}$ TOBB-ETU Hospital, Ankara, Turkey \\ Correspondence should be addressed to Nejat Akar, akar@medicine.ankara.edu.tr
}

Received 6 September 2011; Accepted 5 December 2011

Academic Editor: Helena Kempski

Copyright ( $\odot 2012$ Dilara Fatma Akın et al. This is an open access article distributed under the Creative Commons Attribution License, which permits unrestricted use, distribution, and reproduction in any medium, provided the original work is properly cited.

This study was undertaken to determine the prevalence of the Factor V 1691 G-A and PT 20210 G-A mutations in Turkish children with leukemia. We genotyped 135 pediatric leukemia patients with for these mutations. Eleven (8\%) of the 135 patients were heterozygous for the FV 1691 G-A mutation. Seven (5,1\%) of the patients carried the PT 20210 G-A heterozygous mutation. Of the 135 patients, only three had thrombotic event, none of which had these two mutations, which is common in Turkish population. Our findings revealed a controversial compared to the previous reports, which needs further investigation.

\section{Introduction}

The reported incidence of thrombosis ranges from $2.4 \%$ to $11.5 \%$ and an important complication in pediatric acute lymphoblastic leukaemia (ALL) patients. Its occurrence may complicate the treatment course with a negative prognostic impact $[2,3]$.

Thromboembolic events (TEs) are thought to result from the interaction of various factors, including effects of disease itself, central venous line, and chemotherapy, catheterization, infections, dehydration, chemotherapeutic agents such as steroids and L-asparaginase (L-Asp), and acquired or inherited prothrombotic defects (IPDs) may influence the occurrence of thromboembolism [4-8].

Various molecular defects of different hemostatic components have been established as risk factors for thromboembolic diseases mainly in adults and pediatric cases such as deficiencies of protein $\mathrm{C}$, protein $\mathrm{S}$, and antithrombin, resistance to activated protein $\mathrm{C}$, mostly due to the factor $\mathrm{V}(\mathrm{FV})$ G1691A gene mutation and the prothrombin (PT) G20210A genotype [9].
Chemotherapy can influence the haemostatic system either through the direct effect of the chemotherapeutic agent or through complications such as infections. Corticosteroids activate platelet function, asparaginase reduces the synthesis of natural anticoagulants and in combination they increase the risk of TE in children with ALL. Some studies have shown increased thrombin activation at diagnosis in children with ALL $[6,9]$.

Factor V gene G-A transition at nucleotide 1691 in exon 10 is the leading cause of constitutional thrombophilia and results in with thrombus formation and creates a protein that is resistant to APC in the majority of affected individuals. The risk of developing thrombotic episodes for persons heterozygous for the FVL mutation is about 5-10 fold and for those homozygous is $80-100$-fold higher than the general population. The frequency of FVL is about $8 \%$ in our healthy population [10].

The prothrombin $20210 \mathrm{G}-\mathrm{A}$ polymorphism is the second most common inherited risk factor for thrombophilia. The polymorphism is located at position 20210 in the $3^{\prime}$ untranslated region of the prothrombin gene and is caused 
by single base change G-A. Carriers of the rare A allele have higher prothrombin levels than carriers of the $G$ allele and a higher risk of venous thrombosis $[11,12]$. The frequency of PT 20210 is $2.6 \%$ in our healthy population [13].

Several studies on genetic risk factors for thrombosis excluded those patients with cancer-related thrombosis. Although surgery, chemotherapy, central venous catheters, and systemic hypercoagulability were associated with venous thromboembolism (VTE) in cancer, previous reports on the association of FVL and/or prothrombin 20210A with cancerassociated VTE are few and present conflicting results [14$18]$.

Recent studies reported that the Factor V Leiden mutation does not play a significant role on the overall incidence of thrombosis that occurs in children with cancer. The North American Prophylactic Antithrombin Replacement in Kids with Acute Lymphoblastic Leukemia Treated with Asparaginase (PARKAA) study failed to show any correlation between the presence of the PT 20210A variant or the FV G1691A mutation and development of asymptomatic VTE [15]. A study by Mitchell et al. reported that none of 22 children with TEs were positive for factor $\mathrm{V}$ Leiden or prothrombin gene 20201A but 22 of 60 children had TEs, a prevalence of $36.7 \%$ without this mutation [19].

Nowak-Göttl et al. reported in a prospective multicenter study which focused on the role of prothrombotic risk factors in consecutively admitted newly diagnosed children with ALL carrying prothrombotic risk factors. In this study 32 of 289 consecutively admitted leukemic children $(11 \%)$ treated according to the ALL-BFM 90/95 study suffered venous thromboembolism [20]. Caruso et al. demonstrated that inherited thrombophilia increased the risk of VTE in pediatric ALL patients by approximately $8.5(4.4-17.4)$. They indicated that a meta-analysis of prospective studies in childhood ALL and VTE found that symptomatic VTE was diagnosed in the central nervous system (CNS) in 50\% of cases, venous VTE in $31 \%$, and cerebral infarction or stroke in $18 \%$ [3].

As several previous reports had conflicting data on common thrombophilic mutations in leukemic children, we screened our patients with pediatric leukemia for these two mutations.

\section{Patients and Methods}

This study population consisted of 135 patients 78 males and 57 females, aged between 1 and 15 years who were admitted to our hospital with the diagnosis of pediatric leukemia.114 had ALL, 13 had AML, 6 had chronic myelocyctic leukemia (CML), and 1 patient had mixed leukemia, 1 patient acute promyelocytic leukemia (APL).

An informed written consent was obtained from all the patients' parents. Blood samples were collected with EDTAcontaining tubes and DNA was extracted from peripheral blood leukocytes according to phenol-chloroform method. FV 1691 G-A and PT 20210 G-A mutation was analysed by real-time PCR (RT-PCR).

Genotyping of FV 1691 G-A, PT 20210 G-AT polymorphisms was screened with real-time PCR using fluorescence melting curve detection analysis by means of the Light Cycler
System (Roche Diagnostics, Manheim, Germany). For FV 1691 G-A, forward primer: 5' ${ }^{\prime}$ TGCCCAGTGCTTAACAAGACCA-3'; reverse primer: 5' -CTTGAAGGAAATGCCCCATTA-3'; anchor hybridization probe: $5^{\prime}$-LC-Red705TGTCCTTGAAGTAACCTTTCAGAAATTCTG-3'-PHO; mutation probe: $5^{\prime}$-GGCGAGGAATACAGGTAT-3'. For prothrombin, forward primer: 5'-CCGCTGGTATCAAATGGGG-3'; reverse primer: $5^{\prime}$-CCAGTAGTATTACTGGCTCTTCCTG-3' ${ }^{\prime}$, anchor hybridization probe: $5^{\prime}$-LC-Red640TCCCAGTGCTATTCATGGGC-3'-PHO; mutation probe: 5'-CTCAGCGAGCCTCAATG- $3^{\prime}$.

Melting point analysis was performed according to the instructions of the company.

\section{Statistical Analysis}

The chi-square test was used to compare categorical variables. $P$ value of $<.05$ was considered statistically significant. Allelic frequencies were calculated by gene-counting method and the genotype distribution with Hardy-Weinberg expectations by a $x^{2}$ and Fisher's exact tests.

\section{Results}

Genotype distributions of FV 1691 G-A, PT 20210 G-A were given in Table 1, respectively.

Eleven of the 135 patients were heterozygous for the FV 1691 G-A mutation. Seven of the patients carried the PT 20210 G-A heterozygous mutation. FV 1691A homozygous mutant genotype was found in 3 of the 135 patients and homozygous PT 20210A mutant genotype was not detected in 135 patients. None of the patients carried both mutations. In childhood leukemia patients group allelic distributions for FV $1691 \mathrm{G}$ is $0,93 \%$, A allele is $0,06 \%$ and PT $20210 \mathrm{G}$ is $0,97 \%$ and A allele is $0,02 \%$. Heterozygosities of FV $1691 \mathrm{G}-$ A substitution demonstrate a difference between childhood leukemia patients and healthy newborns [(OR: 1.25/0.6-2,4 CI (95\%); $P: 0,03)$ ] (Table 2).

Three $(2,2 \%)$ of the 135 patients had thromboembolism. Sinus thrombosis, the cerebral ischemia, and deep vein thrombosis were the diagnosis of these cases. Neither of these three patients had thrombophilic mutations.

\section{Discussion}

This study was undertaken to determine the prevalence of the Factor V 1691 G-A and PT 20210 G-A mutations in Turkish children with leukemia. These two mutations have been shown to be the most common heritable risk factor predisposing to venous thrombosis in adults [21-23] and also in the Turkish population $[10,24]$.

In this study, we have shown that Factor $1691 \mathrm{G}-\mathrm{A}$ and PT 20210 G-A mutations are not associated with the development of thrombosis in our childhood leukemia patients. Other studies reported controversial data on PT than FV mutations $[9,14,15]$. The prevalence and the pathogenesis of thrombosis associated with ALL are obscure. The primary disease itself can activate blood coagulation via procoagulant 
TABLE 1: Genotype distributions in patients for FV 1691 G-A and PT 20210 G-A.

\begin{tabular}{lccc}
\hline Polymorphism FV 1691 G-A & Genotype distributions $n: 135(\%)$ & Polymorphism PT 20210 G-A & Genotype distributions $n: 135(\%)$ \\
\hline G/G & $121(89,6 \%)$ & G/G & $128(94,8 \%)$ \\
G/A & $11(8 \%)$ & G/A & $7(5,1 \%)$ \\
A/A & $3(2 \%)$ & A/A & - \\
\hline
\end{tabular}

TABLE 2: Comparison of FV 1691 G-A in between childhood leukemia and healthy newborns gruops.

\begin{tabular}{lccc}
\hline Polymorphism FV 1691 G-A & Childhood leukemia $n: 135(\%)$ & Newborns* $n: 551(\%)$ & OR CI (95\%) \\
\hline G/G & $121(89,6 \%)$ & $494(89,6)$ & 1 \\
G/A & $11(8 \%)$ & $56(10,1 \%)$ & $1,25(0,6-2,4)$ \\
A/A & $3(2 \%)$ & $1(0,1 \%)$ & 0,5 \\
\hline
\end{tabular}

Reference: *[1].

substances or by impairment of fibrinolytic or anticoagulant pathways. Additionally, chemotherapy and prothrombotic risk factors of the host may play a contributory role. Epidemiologic studies of pediatric thrombosis in leukemia have been greatly emphasized by small numbers, making estimates of thrombosis risk in this condition very difficult $[3,14,25$, 26]. The preponderance of the data, including ours, suggests no association of FVL and PT 20210A with leukemia-related thrombosis. Anticoagulant therapy initiated to all patients, which may be the reason for low prevelence of thrombosis.

In conclusion, our study suggests that the FV 1691A and the Prothrombin 20210A mutations are not associated with $\mathrm{TE}$ in pediatric leukemias.

\section{References}

[1] N. Akar, "Factor V Leiden and Natural Selection," Clinical and Applied Thrombosis/Hemostasis. In press.

[2] U. H. Athale and A. K. C. Chan, "Thrombosis in children with acute lymphoblastic leukemia: Part I. Epidemiology of thrombosis in children with acute lymphoblastic leukemia," Thrombosis Research, vol. 111, no. 3, pp. 125-131, 2003.

[3] V. Caruso, L. Iacoviello, A. Di Castelnuovo et al., "Thrombotic complications in childhood acute lymphoblastic leukemia: a meta-analysis of 17 prospective studies comprising 1752 pediatric patients," Blood, vol. 108, no. 7, pp. 2216-2222, 2006.

[4] N. Santoro, P. Giordano, G. C. Del Vecchio et al., "Ischemic stroke in children treated for acute lymphoblastic leukemia: a retrospective study," Journal of Pediatric Hematology/Oncology, vol. 27, no. 3, pp. 153-157, 2005.

[5] F. R. Rickles and A. Falanga, "Molecular basis for the relationship between thrombosis and cancer," Thrombosis Research, vol. 102, no. 6, pp. V215-V224, 2001.

[6] D. E. Sutherland, I. C. Weitz, and H. A. Liebman, "Thromboembolic complications of cancer: epidemiology, pathogenesis, diagnosis, and treatment," American Journal of Hematology, vol. 72, no. 1, pp. 43-52, 2003.

[7] P. Giordano, G. C. Del Vecchio, P. Saracco et al., "A practical approach to diagnosis and treatment of symptomatic thromboembolic events in children with acute lymphoblastic leukemia: recommendations of the coagulation defects" AIEOP Working Group," Recent Patents on Cardiovascular Drug Discovery, vol. 2, no. 1, pp. 53-62, 2007.
[8] T. Celkan, H. Apak, A. Ozkan et al., "The etiology of thromboembolism in hospitalized children," Turkish Pediatric Archive, vol. 39, pp. 65-70, 2004.

[9] U. Nowak-Göttl, R. Junker, W. Kreuz et al., "Risk of recurrent venous thrombosis in children with combined prothrombotic risk factors," Blood, vol. 97, no. 4, pp. 858-862, 2001.

[10] N. Akar, "Factor V 1691 G-A mutation distribution in a healthy Turkish population," Turkish Journal of Hematology, vol. 26, no. 1, pp. 9-11, 2009.

[11] E. M. Bladbjerg, K. Andersen-Ranberg, M. P.M. De Maat et al., "Longevity is independent of common variations in genes associated with cardiovascular risk," Thrombosis and Haemostasis, vol. 82, no. 3, pp. 1100-1105, 1999.

[12] C. J. M. Doggen, V. M. Cats, R. M. Bertina, and F. R. Rosendaal, "Interaction of coagulation defects and cardiovascular risk factors: increased risk of myocardial infarction associated with factor V Leiden or prothrombin 20210A," Circulation, vol. 97, no. 11, pp. 1037-1041, 1998.

[13] N. Akar and M. Misirlioglu, "Prothrombin gene 20210 G-A mutation in the turkish population," American Journal of Hematology, vol. 58, no. 3, p. 249, 1998.

[14] H. M. M. B. Otten, J. Mathijssen, H. Ten Cate et al., "Symptomatic venous thromboembolism in cancer patients treated with chemotherapy: an underestimated phenomenon," Archives of Internal Medicine, vol. 164, no. 2, pp. 190-194, 2004.

[15] M. T. Sifontes, R. Nuss, S. P. Hunger, J. Wilimas, L. J. Jacobson, and M. J. Manco-Johnson, "The factor V Leiden mutation in children with cancer and thrombosis," British Journal of Haematology, vol. 96, no. 3, pp. 484-489, 1997.

[16] N. Haim, N. Lanir, R. Hoffman, A. Haim, M. Tsalik, and B. Brenner, "Acquired activated protein $\mathrm{C}$ resistance is common in cancer patients and is associated with venous thromboembolism," American Journal of Medicine, vol. 110, no. 2, pp. 9196, 2001.

[17] R. Pihusch, G. Danzl, M. Scholz et al., "Impact of thrombophilic gene mutations on thrombosis risk in patients with gastrointestinal carcinoma," Cancer, vol. 94, no. 12, pp. 31203126, 2002.

[18] E. Ramacciotti, N. Wolosker, P. Puech-Leao et al., "Prevalence of factor V Leiden, FII G20210A, FXIII Val34Leu and MTHFR C677T polymorphisms in cancer patients with and without venous thrombosis," Thrombosis Research, vol. 109, no. 4, pp. 171-174, 2003.

[19] L. G. Mitchell, M. Andrew, T. Abshire et al., "A prospective cohort study determining the prevalence of thrombotic events 
in children with acute lymphoblastic leukemia and a central venous line who are treated with L-asparaginase: results of the Prophylactic Antithrombin Replacement in Kids with Acute Lymphoblastic Leukemia Treated with Asparaginase (PARKAA) Study," Cancer, vol. 97, no. 2, pp. 508-516, 2003.

[20] U. Nowak-Göttl, G. Kenet, and L. G. Mitchell, "Thrombosis in childhood acute lymphoblastic leukaemia: epidemiology, aetiology, diagnosis, prevention and treatment," Best Practice and Research: Clinical Haematology, vol. 22, no. 1, pp. 103-114, 2009.

[21] B. Dahlback, M. Carlsson, and P. J. Svensson, "Familial thrombophilia due to a previously unrecognized mechanism characterized by poor anticoagulant response to activated protein C: prediction of a cofactor to activated protein C," Proceedings of the National Academy of Sciences of the United States of America, vol. 90, no. 3, pp. 1004-1008, 1993.

[22] T. Koster, F. R. Rosendaal, H. De Ronde, E. Briet, J. P. Vandenbroucke, and R. M. Bertina, "Venous thrombosis due to poor anticoagulant response to activated protein C: Leiden Thrombophilia Study," Lancet, vol. 342, no. 8886-8887, pp. 15031506, 1993.

[23] J. S. Greengard, S. Eichinger, J. H. Griffin, and K. A. Bauer, "Brief report: variability of thrombosis among homozygous siblings with resistance to activated protein C due to an Arg $\rightarrow$ Gln mutation in the gene for factor V," New England Journal of Medicine, vol. 331, no. 23, pp. 1559-1562, 1994.

[24] A. Eroglu, D. Sertkaya, and N. Akar, "The fole of factor V Leiden in adult patients with venous thromboembolism: a meta-analysis of published studies from Turkey," Clinical and Applied Thrombosis/Hemostasis. In press.

[25] M. Calık, I. E. Piskin, G. Utsuda, and H. Kardes, "A case of acute myeloid leukemia presenting with deep venous thrombosis," Journal of Clinical and Experimental Investigations, vol. 2, no. 1, pp. 114-117, 2011

[26] S. Akarsu, E. Yılmaz, D. E. Aygün, and A. Gozdasoglu, "Lasparaginase therapy and complications in children with acute lymphoblastic leukemia," Turkish Pediatric Archive, vol. 39, pp. 162-170, 2004. 


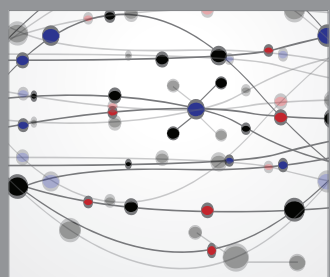

The Scientific World Journal
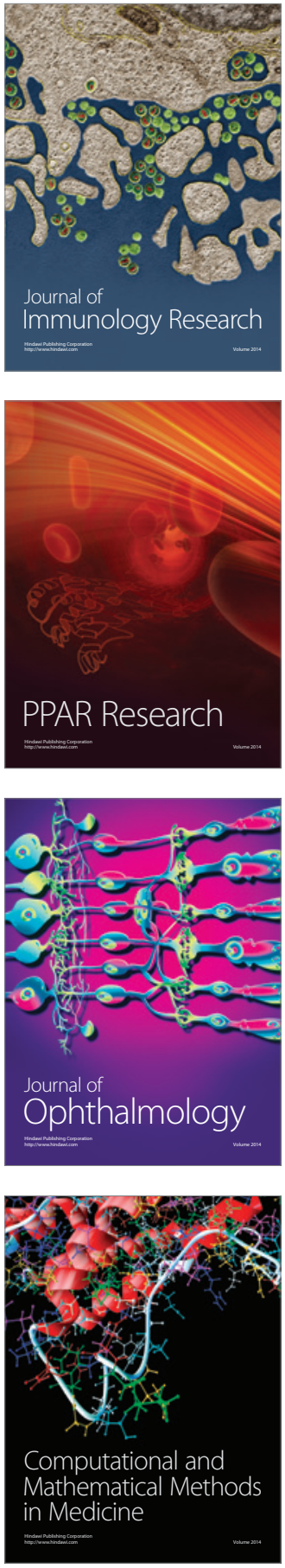

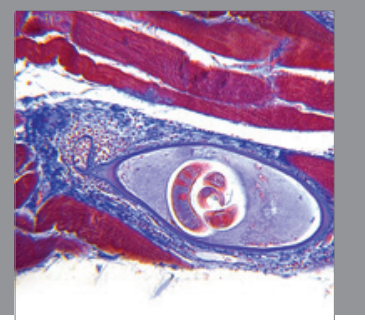

Gastroenterology

Research and Practice
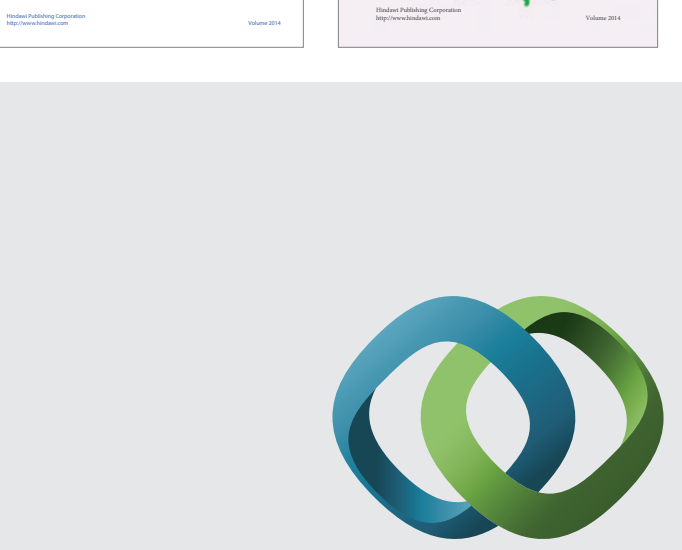

\section{Hindawi}

Submit your manuscripts at

http://www.hindawi.com
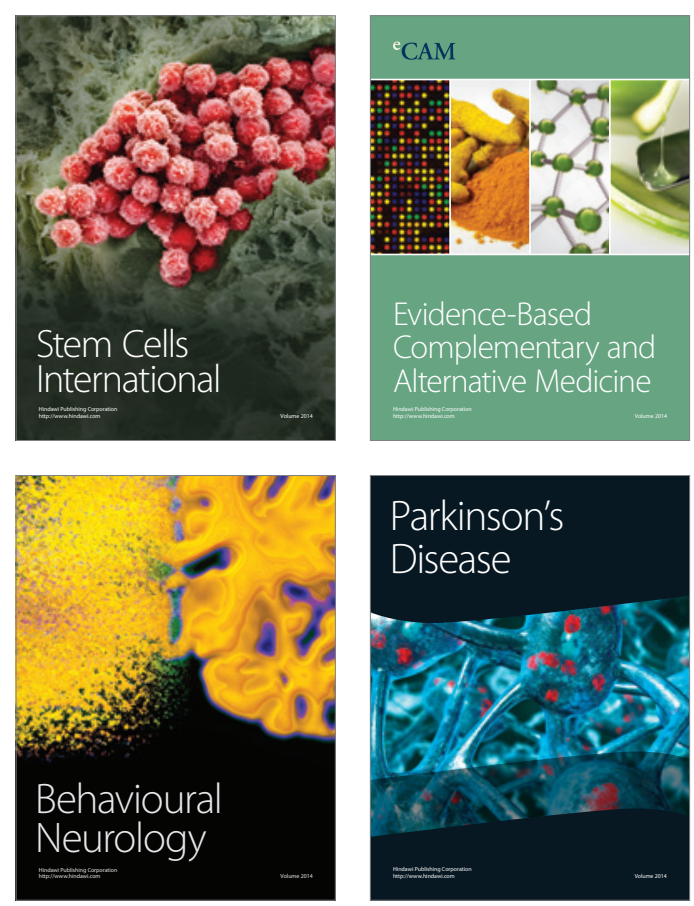

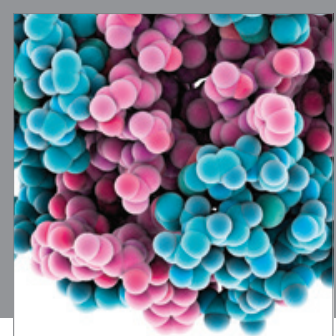

Journal of
Diabetes Research

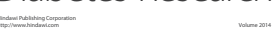

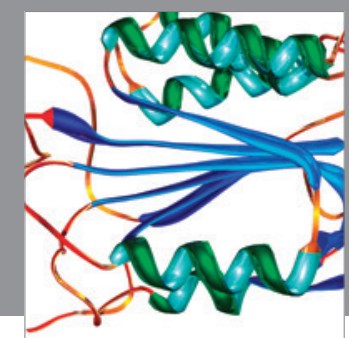

Disease Markers
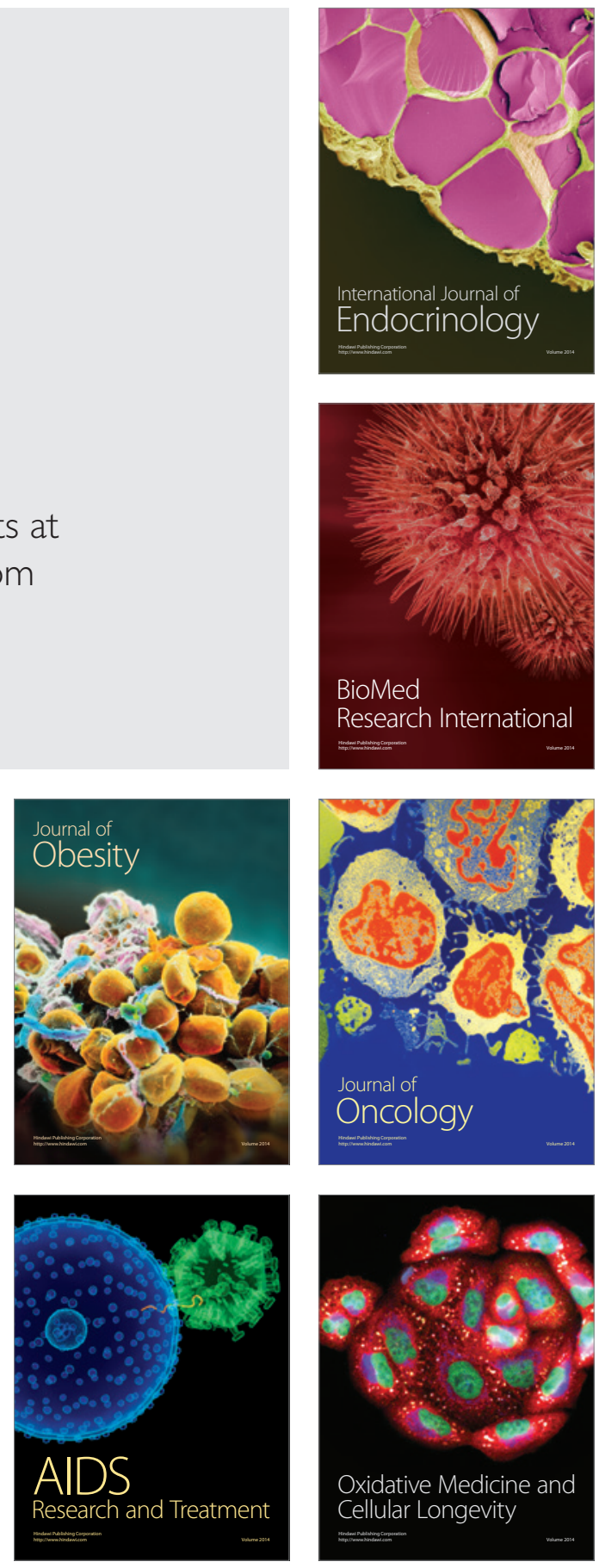